\title{
Treatable inborn errors of metabolism presenting as cerebral palsy mimics: systematic literature review
}

Emma L Leach ${ }^{1,2}$, Michael Shevell ${ }^{3,4}$, Kristin Bowden², Sylvia Stockler-Ipsiroglu²,5,6 and Clara DM van Karnebeek ${ }^{2,5,6,7,8^{*}}$

\begin{abstract}
Background: Inborn errors of metabolism (IEMs) have been anecdotally reported in the literature as presenting with features of cerebral palsy (CP) or misdiagnosed as 'atypical CP'. A significant proportion is amenable to treatment either directly targeting the underlying pathophysiology (often with improvement of symptoms) or with the potential to halt disease progression and prevent/minimize further damage.
\end{abstract}

Methods: We performed a systematic literature review to identify all reports of IEMs presenting with CP-like symptoms before 5 years of age, and selected those for which evidence for effective treatment exists.

Results: We identified 54 treatable IEMs reported to mimic CP, belonging to 13 different biochemical categories. A further 13 treatable IEMs were included, which can present with CP-like symptoms according to expert opinion, but for which no reports in the literature were identified. For 26 of these IEMs, a treatment is available that targets the primary underlying pathophysiology (e.g. neurotransmitter supplements), and for the remainder $(n=41)$ treatment exerts stabilizing/preventative effects (e.g. emergency regimen). The total number of treatments is 50 , and evidence varies for the various treatments from Level 1b, c $(n=2)$; Level 2a, b, c $(n=16)$; Level $4(n=35)$; to Level 4-5 $(n=6)$; Level $5(n=8)$. Thirty-eight $(57 \%)$ of the treatable IEMs mimicking CP can be identified by ready available metabolic screening tests in blood or urine, while the remaining IEMs require more specific and sometimes invasive tests.

Conclusions: Limited by the rare nature of IEMs and incomplete information in the literature, we conclude that (1) A surprisingly large number of IEMs can present with CP symptoms, as 'CP mimics', (2) although individually rare, a large proportion of these diseases are treatable such that neurological damage can either be reversed or prevented, (3) clinician awareness of treatable CP mimics is important for appropriate screening, diagnosis, and early intervention, and (4) systematic studies are required to elucidate the collective frequency of treatable IEMs in CP.

Keywords: Inherited metabolic diseases, Therapy, Diagnosis, Atypical cerebral palsy, Movement disorders

\section{Background}

Cerebral palsy (CP) is defined as a group of nonprogressive disorders of movement and posture, which cause activity limitations due to disturbances that occurred in the developing fetal or infant brain [1]. CP is the most common cause of physical impairment in the pediatric population with a prevalence of $2-3$ per 1000 live births $[2,3]$. Risk factors include prematurity, kernicterus/ hyperbilirubinemia, early CNS infection, non-specific fetal

\footnotetext{
* Correspondence: cvankarnebeek@cw.bc.ca

2Division of Biochemical Diseases, Department of Pediatrics, BC Children's Hospital, University of British Columbia, Vancouver, Canada

${ }^{5}$ Division of Biochemical Diseases, Department of Pediatrics, BC Children's Hospital, Vancouver, Canada

Full list of author information is available at the end of the article
}

or maternal infection, intra-partum asphyxia, birth trauma and intracranial hemorrhage or neonatal encephalitis [4]. However, despite advances in maternal care and obstetrical intervention in recent decades, the incidence of $\mathrm{CP}$ has not declined [5]. Characterization of CP is traditionally based on the predominant quality of motor impairment (spastic, dyskinetic, ataxic-hypotonic or mixed) [6], assessed on standard neurologic examination.

$\mathrm{CP}$ is frequently associated with cognitive, behavioral, and sensory impairments as well as epilepsy [7]. The most common morbidity, noted in approximately $40-65 \%$ of all children with CP, is intellectual developmental disability (IDD), defined by significant delay in two or more 
developmental domains at age less than 5 years and an intelligence quotient of $\leq 70$ at older age. [8]. Children with co-occurring ID are at increased risk of emotional and behavioral problems [9] and other chronic health conditions requiring frequent hospitalizations $[10,11]$ with a high burden of care and utilization of health services for individuals with $\mathrm{CP}$ and their families [7]. The associated medical expenditures are considerable; Kancerla et al. [12] showed that annual costs for children with CP exceed those of children without CP by $\$ 15,047$ USD and in case of co-occurring ID by $\$ 26,617$ USD. Treatment and ultimately prevention of CP (and IDD) therefore is essential to reduce the emotional and physical suffering of patients and families, and to reduce the immense health care costs.

Determination of the underlying cause of $\mathrm{CP}$, whether due to a malformation, injury acquired during the pre-, peri-, or postnatal period, or a genetic aberration has obvious significance from the point of view of assessment of risk, counseling of families, and developing prevention and intervention strategies [13]. The implicit heterogeneity of CP poses a challenge for diagnosis and treatment [14], and the current management of CP follows a symptomatic approach (e.g., baclofen to relieve spasticity; occupational therapy to improve mobility; pain management).

However, there are reports in the literature of inborn errors of metabolism (IEMs) that present as CP mimics, many of which are in fact amenable to therapy targeting the underlying cause that can improve neurological outcomes. IEMs are a collection of rare genetic diseases that generally result from a deficiency of an intracellular component (e.g., an enzyme or transporter) of a metabolic pathway, resulting in an accumulation of a substrate or intermediate in a pathway and/or reduced ability to synthesize essential compounds. Often the central nervous system (CNS) is affected, leading to neurological disease [15]. An example is Segawa disease, also called GTPCH1deficient dopa-responsive dystonia (GTPCH1-DRD), characterized by dystonia in childhood that is often misdiagnosed as CP e.g., [16,17]. This neurotransmitter disorder can be diagnosed by standard analysis of neurotransmitter metabolites in the cerebrospinal fluid. Individuals with GTPCH1-DRD benefit from treatment with BH4 [18], amine replacement, as well as levodopa. The majority of treated individuals shows rapid clinical improvement in both CP-related symptoms (spasticity, dystonia, general tone) and are able to lead "an entirely normal life" [16].

It is currently unknown how many such treatable $\mathrm{CP}$-mimics exist, as the evidence has not been systematically reviewed. We model the current review after our Treatable Intellectual Disability Endeavor (TIDE) study, which published a list of 89 treatable IEMs that present with an IDD [19], and a diagnostic algorithm with App [20]. This algorithm has been implemented in more than 400 children with unexplained IDD as part of the TIDE-BC study at the British Columbia Children's Hospital, in Vancouver, Canada, and treatable IEMs were identified in more than 5\% [20], which serves as motivation for the work presented here.

\section{Methods}

We performed the first systematic review to compile evidence from the literature and clinical expertise of all IEMs that are known to present with CP symptoms, with a focus on those that are amenable to causal treatment. We aim to raise awareness of existence of CP mimics and formulate a diagnostic algorithm to support clinicians in the effective identification of these IEMs. We followed the Preferred Reporting Items for Systematic Reviews and Meta-Analyses (PRISMA) statement (http:// www.prisma-statement.org/) [21].

\section{Information sources}

A systematic review was performed to identify all reports of inborn errors of metabolism (IEMs) presenting with CP symptoms by searching the PubMed database, checking reference lists of relevant articles and consulting with experts in the field. We considered only articles that were published in English, described findings in humans, and those where full text publication was available electronically through our institution's subscription. No publication date restrictions were imposed and articles included in analyses were published between 1957-2014. The last search was performed October 15, 2014.

\section{Definitions \& Eligibility criteria}

We included only studies describing diseases that are IEMs, which we have previously defined as "genetic disease involving a disorder of metabolism with confirmation based on the internationally accepted diagnostic test(s) for that IEM (gene mutations, enzyme deficiency, or specific biochemical marker); this term excludes endocrine disorders" [19]. To identify diseases most likely to be misdiagnosed as CP (i.e., to exclude adult-onset), we limited our inclusion to reports where CP symptoms (Table 1) present before 5 years of age in at least one reported case.

The goal of this systematic review was to identify all treatable IEMs that present with CP symptoms, rather than to identify every report. We selected the most reliable or comprehensive reference for this publication; this implies that additional case reports of CP symptoms for a given IEM beyond those cited here may exist.

\section{Search strategy}

The search strategy is outlined in Table 1. Primarily, combinations of search terms that describe cerebral palsy symptoms and inborn errors of metabolism were used to identify relevant articles in the PubMed database 
Table 1 Search strategy to systematically review treatable IEMs that present as CP mimics

\begin{tabular}{|c|c|c|c|}
\hline \multicolumn{3}{|l|}{ Search strategy } & \multirow{2}{*}{$\frac{\text { \# Treatable CP mimics }}{27}$} \\
\hline 1) PubMed search using combinations of & Definition: & Search terms: & \\
\hline $\begin{array}{l}\text { alternative terms to describe the CP } \\
\text { phenotype and IEMs }\end{array}$ & Cerebral palsy & $\begin{array}{l}\text { Cerebral palsy, spastic, spasticity, dystonia, } \\
\text { dyskinesia, ataxia, movement disorder, gait } \\
\text { abnormalities, hypoxic(+/-ischemic) } \\
\text { encephalopathy, perinatal asphyxia }\end{array}$ & \\
\hline & Inborn error of metabolism & $\begin{array}{l}\text { Inborn error of metabolism, metabolic } \\
\text { disease, amino acids, creatine, mitochondria, } \\
\text { neurotransmitter, organic acid, urea cycle, vitamins }\end{array}$ & \\
\hline 2) Manual search & \multicolumn{2}{|c|}{$\begin{array}{l}\text { Reference lists of articles identified through PubMed } \\
\text { search were manually screened for additional citations. }\end{array}$} & 15 \\
\hline $\begin{array}{l}\text { 3) Targeted search of known treatable } \\
\text { IEMs causing ID }\end{array}$ & \multicolumn{2}{|c|}{$\begin{array}{l}\text { Diseases listed in the TIDE App (www.treatable-id.org) as } \\
\text { treatable IEMs searched in combination with the above IEM search terms. }\end{array}$} & 12 \\
\hline \multicolumn{3}{|l|}{ Total number of IEMs identified } & 54 \\
\hline
\end{tabular}

(see Table 1). Different combinations yielded common articles, indicating our approach is conservative and our search terms are effective. Results from all key word combination searches were pooled and sorted to identify 472 unique articles (i.e., without duplicate results), which were then manually screened based on inclusion/ exclusion criteria by the first author (ELL), with duplicate publications and those that did not meet eligibility criteria being removed. As well, reference lists of these publications were screened for additional relevant articles and these were judged for inclusion using the same criteria. Finally, to ensure that we have identified all salient IEMs, we performed another search using known treatable IEMs listed in a previous publication of treatable IEMs that present with intellectual disability (ID) [19] in combination with our search terms describing CP. Initial searches were performed independently by ELL following review protocol and all possible studies were compiled into a table. The generated list of IEMs was reviewed periodically by experts SS and CvK to confirm data, refine the search strategy and inclusion criteria.

\section{Data collection}

The following information was extracted from each article: disease name, cerebral palsy symptoms as reported by the original authors, age of symptom onset, any treatment used (with the dose and frequency) and the treatment outcome as described by the authors. For completeness, we later assigned each disease to a biochemical category and retrieved OMIM identifiers, underlying genes and pattern of inheritance, appropriate diagnostic test, and standard of care treatment; all were agreed upon by expert clinicians. Diagnostic tests were compared with a recent diagnostic algorithm of treatable IEMs [20] and with accepted clinical practices (e.g., GeneReviews ${ }^{\oplus}$ ). Given the positive experience with the 2-tiered protocol for the work-up the child with IDD for treatable IEMs, we have used this as a basis for the evaluation of the child with CP for similar conditions.

\section{Characterizing treatment \& effects}

As most reviewed studies were case reports, the type of outcome measures varied. We noted whether or not treatment was implemented and the original author's observations on treatment effect. However, due to the time span of the reviewed publications, some treatments are now available that were not at the time of the original publication. Therefore, we took a more objective approach of employing clinical expertise to determine the standard of care treatment for the systematically identified CP mimics and categorized them as treatable versus non-treatable. Causal treatment for this proportion of conditions has been thoroughly evaluated by us already [19], and we apply the same treatment recommendations here. For conditions where treatment was not previously reviewed by us, we adhered to guidelines for the specific IEMs and where needed provided expert input, with consideration for treatments applied in the original case reports of CP mimics.

Treatment of IEMs can be either 'primary treatment', which targets the pathophysiology at a cellular level and improves at least the CP features (muscle tone, rigidity, etc.) plus/minus the cognitive, behavioural, and MRI features; or 'stabilizing/preventative treatment', which halts decline and/or prevents further damage, especially during metabolic crises. For example, creatine supplementation in creatine deficiencies targets the underlying cause of the IEM and can reverse the features. Several disorders caused by deficiencies in vitamins or co-factors can show improvement in primary features with appropriate supplementation. Examples of stabilizing/preventative treatment include emergency regimen for fatty acid oxidation disorders, HSCT for lysosomal disorders and dietary restriction 
of amino acids in hyperhomocysteinemias and amnio acid disorders.

Non-treatable IEMs are those for which treatment of the underlying cause is not available (i.e., only treatment of symptoms) or has shown to not be consistently effective. We summarize currently non-treatable IEMs in the hope that when treatment does become available, clinicians will be aware that these diseases can mimic $\mathrm{CP}$ and can then intervene.

\section{Levels of evidence}

Levels of evidence for treatments were evaluated based on existing level classification by the Centre of EvidenceBased Medicine (www.cebm.net): Level 1a=Systematic Review of RCTs, $1 b=$ Individual RCT, $1 \mathrm{c}=$ 'All or None' [ $=$ (prolongation of) survival with therapy]; Level $2 \mathrm{a}=$ Systematic Review of Cohort Studies, $2 \mathrm{~b}=$ Individual Cohort Study, 2c = 'Outcomes Research' [focussed on end results of therapy for chronic conditions, including functioning and quality of life]; Level 3 = Systematic Review of CaseControl Studies; Level 4=Individual Case-Control Study or Case-series/report; Level $5=$ Expert opinion without critical appraisal; based on physiology, bench research or first principles.

\section{Expert-identified CP mimics}

The authors who are expert clinicians in pediatric IEMs (CvK \& SS) identified 13 additional diseases which they have designated as $\mathrm{CP}$ mimics in their clinical experience. For the majority of these, the pathophysiology is identical to IEMs in the same category for which literature evidence does exist; for example PSPH and PSAT deficiency which are both characterized by lack of serine in the brain similar to PGHDH deficiency (primarily treatable by serine supplements), or urea cycle defects in which hyperammonemic crises cause irreversible brain damage (which are preventable via dietary manipulation and medication), just like OTC deficiency for which CP-like features have been described. Likely such diseases have not (yet) been described to present as $\mathrm{CP}$ in the literature, likely due to the rare frequency and the increasing challenge of publishing case reports alone. The expert opinion approach was used, so that also for these IEMs, affected individuals might also receive the benefit of early detection and intervention.

\section{Diagnostic algorithm}

To provide guidance for the identification of treatable CP-mimics, we used the 2-tiered algorithm published by van Karnebeek et al. [19] to identify treatable IEMs in children presenting with IDD: First tier tests are generally accessible and offered by most biochemical genetics laboratories around the world with reasonable turn-around times and affordable prices (total costs \$567.97 CAD), including tests in blood (lactate; ammonia; copper; ceruloplasmin; plasma total homocysteine; plasma aminoacids, and bloodspot quantitative acylcarnitine profile) and in urine (creatine metabolites; purines and pyrimidines; organic acids; oligosaccharides; and glycosaminoglycans). Each of these screening tests has the potential to specifically identify treatable IEMs, which is then often confirmed via molecular and/or enzymatic analysis. The $2^{\text {nd }}$ tier requires a more directed, 'single test per disease' approach based on signs and symptoms. In general these tests are more invasive and more expensive.

\section{Results}

\section{Treatable IEMs}

Based on the defined inclusion/exclusion criteria, we identified 54 treatable IEMs reported to mimic CP (Table 1). These are categorized alphabetically into 13 biochemical categories (Table 2): amino acids $(n=5)$, cerebral glucose transport $(n=1)$, creatine $(n=1)$, fatty acid-related processes $(n=3)$, hyperhomocysteinemia $(n=2)$, lipids $(n=1)$, lysosomal $(n=4)$, metals $(n=2)$, mitochondrial $(n=3)$, neurotransmission $(n=9)$, organic acids $(\mathrm{n}=12)$, urea cycle $(\mathrm{n}=4)$ and vitamins/co-factors $(n=7)$. The CP symptoms for each IEM and corresponding reference are described in Additional file 1: Table S1. A further 13 treatable IEMs were identified by expert clinicians on our team (Table 3) for a total of 67 treatable CP mimics. Treatment modalities included: dietary restriction/supplement, co-factor/-enzyme, vitamin, substrate inhibition, substrate reduction, bone marrow and hematopoietic stem cell transplant, gene therapy. The majority of these treatments are accessible and affordable. The total number of different treatments is 50 , and evidence varies for the various treatments from Level $1 b, c(n=2)$; Level 2a, b, c $(n=16)$; Level $4(n=35)$; to Level 4-5 (n=6); Level $5(n=8)$. For $26(39 \%)$ of these IEMs, a treatment is available that targets the primary underlying pathophysiology with the potential to improve $\mathrm{CP}$ symptoms; while for the remaining 41 (61\%) IEMs, treatment is available that stabilizes disease or prevents further damage (e.g., treatment of Succinic semi-aldehyde dehydrogenase deficiency (SSADH) with Vigabatrin can stabilize symptoms $[22,23])$.

Thirty-eight of the 67 disorders $(57 \%)$ of the treatable IEMs described in this review can be identified by ' 1 st tier' metabolic screening tests in blood or urine (Table 4). The other 29 (43\%) require more specific and sometimes invasive methods (' 2 nd $t$ tier tests'; Table 5). Of the $1^{\text {st }}$ tier tests in the Treatable IDD protocol, urine MPS and urine oligosaccharides are not required for the diagnostic evaluation of CP for treatable IEMs. Most $1^{\text {st }}$ tier tests which detect treatable IEMs described in the literature as CP mimics can also identify 'treatable IEMs identified by 
Table 2 Overview of all 54 treatable IEMs presenting as CP mimics identified through systematic literature review

\begin{tabular}{|c|c|c|c|c|c|c|c|}
\hline $\begin{array}{l}\text { Biochemical } \\
\text { category }\end{array}$ & Disease name & OMIM\# & Gene(s) & Treatment & Effect & $\begin{array}{l}\text { Level of } \\
\text { evidence }\end{array}$ & CP symptoms \\
\hline \multirow[t]{5}{*}{ Amino acids } & Hartnup disease & 234500 & SLC6A19 (AR) & High protein diet & $\begin{array}{l}\text { Stabilizing/preventative } \\
\text { treatment }\end{array}$ & $4-5$ & Dystonia \\
\hline & $\begin{array}{l}\text { Hyperornithinemia- } \\
\text { hyperammonemia- } \\
\text { homocitrullinuria }(\mathrm{HHH}) \\
\text { syndrome }\end{array}$ & 238970 & SLC25A15 (AR) & $\begin{array}{l}\text { Dietary protein restriction, } \\
\text { ornithine supplement, sodium } \\
\text { benzoate, phenylacetate }\end{array}$ & $\begin{array}{l}\text { Stabilizing/preventative } \\
\text { treatment }\end{array}$ & 4 & Spasticity \\
\hline & $\begin{array}{l}\text { Late onset non-ketotic } \\
\text { hyperglycinemia }\end{array}$ & 605899 & $\begin{array}{l}\text { AMT/GLDC/GCSH } \\
\text { (AR) }\end{array}$ & $\begin{array}{l}\text { Glycine restriction, +/- } \\
\text { sodium benzoate, NMDA } \\
\text { receptor antagonists, other } \\
\text { neuromodulating agents }\end{array}$ & $\begin{array}{l}\text { Stabilizing/preventative } \\
\text { treatment }\end{array}$ & $4-5$ & Spastic diplegia \\
\hline & Phenylketonuria (PKU) & 261600 & PAH (AR) & $\begin{array}{l}\text { Dietary phenylalanine } \\
\text { restriction }+/- \text { amino acid } \\
\text { supplements (BH(4) } \\
\text { supplement) }\end{array}$ & $\begin{array}{l}\text { Stabilizing/preventative } \\
\text { treatment }\end{array}$ & $2 a(4)$ & Spastic diplegia \\
\hline & $\begin{array}{l}\text { PHGDH deficiency } \\
\text { (Serine deficiency) }\end{array}$ & 601815 & PHGDH (AR) & $\begin{array}{l}\text { L-serine \& }+/ \text {-glycine } \\
\text { supplements }\end{array}$ & $\begin{array}{l}\text { Primary/targeting } \\
\text { underlying } \\
\text { pathophysiology }\end{array}$ & 4 & Spastic diplegia/tetraparesis \\
\hline $\begin{array}{l}\text { Cerebral glucose } \\
\text { transport }\end{array}$ & $\begin{array}{l}\text { Blood brain-barrier glucose- } \\
\text { transporter (GLUT1) defect }\end{array}$ & 606777 & $S L C 2 A 1(A R)$ & Ketogenic diet & $\begin{array}{l}\text { Primary/targeting } \\
\text { underlying } \\
\text { pathophysiology }\end{array}$ & 4 & Spasticity, dystonia, ataxia \\
\hline Creatine & GAMT deficiency & 612736 & GAMT (AR) & $\begin{array}{l}\text { Arginine restriction, creatine } \\
\text { \& ornithine supplements }\end{array}$ & $\begin{array}{l}\text { Primary/targeting } \\
\text { underlying } \\
\text { pathophysiology }\end{array}$ & 4 & $\begin{array}{l}\text { Movement disorder: } \\
\text { extrapyramidal signs, athetosis, } \\
\text { \& ataxia }\end{array}$ \\
\hline \multirow[t]{3}{*}{$\begin{array}{l}\text { Fatty acid } \\
\text { oxidation }\end{array}$} & $\begin{array}{l}\text { Medium-chain acyl-coenzyme } \\
\text { A dehydrogenase (MCAD) } \\
\text { deficiency }\end{array}$ & 201450 & $A C A D M(A R)$ & $\begin{array}{l}\text { Emergency regimen, } \\
\text { L-carnitine, avoid fasting }\end{array}$ & $\begin{array}{l}\text { Stabilizing/preventative } \\
\text { treatment }\end{array}$ & $2 a$ & CP symptoms \\
\hline & $\begin{array}{l}\text { Short-chain acyl-CoA } \\
\text { dehydrogenase (SCAD) } \\
\text { deficiency }\end{array}$ & 201470 & ACADS (AR) & $\begin{array}{l}\text { Emergency regimen, } \\
\text { L-carnitine }\end{array}$ & $\begin{array}{l}\text { Stabilizing/preventative } \\
\text { treatment }\end{array}$ & $2 c$ & Spastic diplegia \\
\hline & $\begin{array}{l}\text { Very-long-chain acyl-CoA } \\
\text { dehydrogenase (VLCAD) } \\
\text { deficiency }\end{array}$ & 201475 & $A C A D V L(A R)$ & $\begin{array}{l}\text { Avoidance of fasting, low-fat } \\
\text { diet, Medium Chain } \\
\text { Triglyceride oild }\end{array}$ & $\begin{array}{l}\text { Stabilizing/preventative } \\
\text { treatment }\end{array}$ & 4 & Neonatal asphyxia \\
\hline \multirow[t]{2}{*}{$\begin{array}{l}\text { Hyperhomo- } \\
\text { cysteinemia }\end{array}$} & $\begin{array}{l}\text { Homocystinuria due to } \\
\text { Cystathionine } \beta \text {-synthase } \\
\text { deficiency }\end{array}$ & 236200 & CBS (AR) & $\begin{array}{l}\text { Methionine restriction, +/- } \\
\text { pyridoxine, }+/- \text { betaine }\end{array}$ & $\begin{array}{l}\text { Stabilizing/preventative } \\
\text { treatment }\end{array}$ & $2 c$ & Dystonia \\
\hline & MTHFR deficiency & 236250 & MTHFR (AR) & $\begin{array}{l}\text { Betaine supplements, }+/- \\
\text { folate, carnitine, methionine } \\
\text { supplements }\end{array}$ & $\begin{array}{l}\text { Stabilizing/preventative } \\
\text { treatment }\end{array}$ & 4 & $\begin{array}{l}\text { Ataxic gait, hypotonia, } \\
\text { extrapyramidal movements, } \\
\text { upper motor neuron signs }\end{array}$ \\
\hline Lipids & Abetalipoproteinemia & 200100 & MTTP (AR) & $\begin{array}{l}\text { Low long-chain fatty acid } \\
\text { diet with fat-soluble vitamin } \\
\text { (i.e., A, D, E, K) supplementation }\end{array}$ & $\begin{array}{l}\text { Primary/targeting } \\
\text { underlying } \\
\text { pathophysiology }\end{array}$ & 4 & Ataxia, abnormal gait \\
\hline
\end{tabular}


Table 2 Overview of all 54 treatable IEMs presenting as CP mimics identified through systematic literature review (Continued)

\begin{tabular}{|c|c|c|c|c|c|c|c|}
\hline \multirow[t]{4}{*}{ Lysosomal } & Fucosidosis & 230000 & FUCA1 (AR) & $\begin{array}{l}\text { Haematopoietic stem cell } \\
\text { transplant }\end{array}$ & $\begin{array}{l}\text { Stabilizing/preventative } \\
\text { treatment }\end{array}$ & 5 & $\begin{array}{l}\text { Severe spasticity; spastic } \\
\text { paresis, generalized dystonia }\end{array}$ \\
\hline & Krabbe disease & 245200 & GALC (AR) & $\begin{array}{l}\text { Haematopoietic stem cell } \\
\text { transplant }\end{array}$ & $\begin{array}{l}\text { Stabilizing/preventative } \\
\text { treatment }\end{array}$ & $2 c$ & Progressive spasticity \\
\hline & $\begin{array}{l}\text { Metachromatic leucodystrophy } \\
\text { (MLD) }\end{array}$ & 250100 & ARSA (AR) & $\begin{array}{l}\text { Haematopoietic stem cell } \\
\text { transplant }\end{array}$ & $\begin{array}{l}\text { Stabilizing/preventative } \\
\text { treatment }\end{array}$ & $4-5$ & $\begin{array}{l}\text { Loss of all gross motor } \\
\text { function measured by CP } \\
\text { scale; ataxia }\end{array}$ \\
\hline & Neimann-Pick, type C & 257220 & $N P C 1, N P C 2$ & Miglustat & $\begin{array}{l}\text { Stabilizing/preventative } \\
\text { treatment }\end{array}$ & $1 b$ & $\begin{array}{l}\text { Axial hypotonia, spastic } \\
\text { diparesis, dystonic posturing } \\
\text { of the hands }\end{array}$ \\
\hline \multirow[t]{2}{*}{ Metals } & Menkes Disease & 309400 & ATP7A & Copper histidine & $\begin{array}{l}\text { Stabilizing/preventative } \\
\text { treatment }\end{array}$ & 4 & $\begin{array}{l}\text { Progressive spasticity, } \\
\text { hypotonia }\end{array}$ \\
\hline & Wilson Disease & 277900 & $A T P 7 B$ & $\begin{array}{l}\text { Zinc \& tetrathiomolybdate; } \\
\text { oxcarbazepine }\end{array}$ & $\begin{array}{l}\text { Stabilizing/preventative } \\
\text { treatment }\end{array}$ & $1 b$ & $\begin{array}{l}\text { Neurological symptoms, } \\
\text { dystonia }\end{array}$ \\
\hline \multirow[t]{3}{*}{ Mitochondria } & Coenzyme Q10 deficiency & 607426 & $\begin{array}{l}\text { COQ2, APTX, PDSS1, } \\
\text { PDSS2, CABC1, COQ9 } \\
\text { (most AR) }\end{array}$ & CoQ supplements & $\begin{array}{l}\text { Primary/targeting } \\
\text { underlying } \\
\text { pathophysiology }\end{array}$ & 4 & $\begin{array}{l}\text { Spastic paresis; progressive } \\
\text { ataxia and dystonia }\end{array}$ \\
\hline & MELAS & 540000 & $\begin{array}{l}m t . A 3243 G, m t . G 13513 A \\
(m t D N A)\end{array}$ & Arginine supplements & $\begin{array}{l}\text { Stabilizing/preventative } \\
\text { treatment }\end{array}$ & $4-5$ & $d x . C P$ \\
\hline & $\begin{array}{l}\text { Pyruvate dehydrogenase } \\
\text { deficiency }\end{array}$ & $\begin{array}{l}312170 \\
245348\end{array}$ & $\begin{array}{l}\text { PDHA1 (X-linked } \\
\text { recessive), DLAT (AR), } \\
\text { PDHX (AR) }\end{array}$ & Ketogenic diet \& thiamine & $\begin{array}{l}\text { Primary/targeting } \\
\text { underlying } \\
\text { pathophysiology }\end{array}$ & 4 & Spastic quadriplegia; dystonia \\
\hline \multirow[t]{6}{*}{ Neurotransmission } & $\begin{array}{l}\text { Aromatic-L-amino-acid } \\
\text { decarboxylase deficiency }\end{array}$ & 608643 & $D D C(A R)$ & $\begin{array}{l}\text { MAO inhibitors, B6, } \\
\text { anti-cholinergics, dopa } \\
\text { agonists) }\end{array}$ & $\begin{array}{l}\text { Primary/targeting } \\
\text { underlying } \\
\text { pathophysiology }\end{array}$ & 4 & $\begin{array}{l}\text { Limb dystonia, athetoid } \\
\text { movement }\end{array}$ \\
\hline & $\begin{array}{l}\text { DHPR deficiency (biopterin } \\
\text { deficiency) }\end{array}$ & 261630 & QDPR (AR) & $\begin{array}{l}\text { BH4, diet, amine replacement, } \\
\text { folinic acid }\end{array}$ & $\begin{array}{l}\text { Primary/targeting } \\
\text { underlying } \\
\text { pathophysiology }\end{array}$ & 4 & $\begin{array}{l}\text { Ataxia, gait disorder, peripheral } \\
\text { spasticity }\end{array}$ \\
\hline & $\begin{array}{l}\text { Dopamine transporter } \\
\text { deficiency syndrome }\end{array}$ & 126455 & $S L C 6 A 3$ & $\begin{array}{l}\text { Dopamine antagonist } \\
\text { (Ropinirole) }\end{array}$ & $\begin{array}{l}\text { Primary/targeting } \\
\text { underlying } \\
\text { pathophysiology }\end{array}$ & 4 & $d x . C P$ \\
\hline & $\begin{array}{l}\text { GTPCH1-deficient } \\
\text { dopa-responsive dystonia } \\
\text { (aka Segawa's disease) }\end{array}$ & 233910 & GCH1 (AR) & $\mathrm{BH} 4$, amine replacement & $\begin{array}{l}\text { Primary/targeting } \\
\text { underlying } \\
\text { pathophysiology }\end{array}$ & 4 & dx. CP; spastic diplegia \\
\hline & $\begin{array}{l}\text { PTPS deficiency (biopterin } \\
\text { deficiency) }\end{array}$ & 261640 & PTS (AR) & BH4, diet, amine replacement & $\begin{array}{l}\text { Primary/targeting } \\
\text { underlying } \\
\text { pathophysiology }\end{array}$ & 4 & $\begin{array}{l}\text { Dystonia; spastic extremities; } \\
\text { generalized dystonia, } \\
\text { choreoathetoid arm } \\
\text { movements \& axial hypotonia }\end{array}$ \\
\hline & $\begin{array}{l}\text { Sepiapterin reductase } \\
\text { deficiency }\end{array}$ & 612716 & $S P R(A R)$ & Amine replacement & $\begin{array}{l}\text { Primary/targeting } \\
\text { underlying } \\
\text { pathophysiology }\end{array}$ & 4 & $\begin{array}{l}\text { Limb spasticity, dystonic } \\
\text { signs; "hypotonic cerebral } \\
\text { palsy"; dystonia, axial } \\
\text { hypotonia; misdx. CP }\end{array}$ \\
\hline
\end{tabular}


Table 2 Overview of all 54 treatable IEMs presenting as CP mimics identified through systematic literature review (Continued)

\begin{tabular}{|c|c|c|c|c|c|c|c|}
\hline & $\begin{array}{l}\text { Succinic semialdehyde } \\
\text { dehydrogenase deficiency } \\
\text { (SSADH) }\end{array}$ & 271980 & $A L D H 5 A 1$ (AR) & Vigabatrin & $\begin{array}{l}\text { Stabilizing/preventative } \\
\text { treatment }\end{array}$ & 4 & $\begin{array}{l}\text { Hypotonia, ataxia; gait } \\
\text { clumsiness, dystonia }\end{array}$ \\
\hline & $\begin{array}{l}\text { Tyrosine hydroxylase } \\
\text { deficiency }\end{array}$ & 605407 & $T H(A R)$ & L-dopa substitution & $\begin{array}{l}\text { Primary/targeting } \\
\text { underlying } \\
\text { pathophysiology }\end{array}$ & 4 & Spastic paraplegia/tetraparesis \\
\hline & $\begin{array}{l}\text { Vesicular monoamine } \\
\text { transporter } 2 \text { (VMAT2) }\end{array}$ & 193001 & SLC18A2 & Dopamine aginist & $\begin{array}{l}\text { Primary/targeting } \\
\text { underlying } \\
\text { pathophysiology }\end{array}$ & 4 & Dystonia \\
\hline \multirow[t]{11}{*}{ Organic acids } & $\beta$-Ketothiolase deficiency & 203750 & ACAT1 (AR) & $\begin{array}{l}\text { Avoid fasting, emergency } \\
\text { regimen, protein restriction }\end{array}$ & $\begin{array}{l}\text { Stabilizing/preventative } \\
\text { treatment }\end{array}$ & 5 & Ataxia, diplegia, hypotonia \\
\hline & $\begin{array}{l}\text { 2-Methyl-3-hydroxybutyryl- } \\
\text { CoA dehydrogenase (MHBD) } \\
\text { deficiency }\end{array}$ & 300438 & HSD17B10 (X-linked) & $\begin{array}{l}\text { Avoid fasting, emergency } \\
\text { regimen, isoleucine restricted } \\
\text { diet }\end{array}$ & $\begin{array}{l}\text { Stabilizing/preventative } \\
\text { treatment }\end{array}$ & 5 & $\begin{array}{l}\text { Ataxia, dystonia, choreoathetosis, } \\
\text { spastic di-/tetra-plegia, } \\
\text { hypotonia }\end{array}$ \\
\hline & $\begin{array}{l}\text { 3-Methylcrotonyl-CoA } \\
\text { carboxylase (MCC) deficiency }\end{array}$ & $\begin{array}{l}210200 ; \\
210210\end{array}$ & MCC1/MCC2 (AR) & $\begin{array}{l}\text { Dietary protein restriction; } \\
\text { carnitine, glycine, biotin } \\
\text { supplements; avoid fasting; } \\
\text { emergency regimen }\end{array}$ & $\begin{array}{l}\text { Stabilizing/preventative } \\
\text { treatment }\end{array}$ & 5 & $d x . C P$ \\
\hline & $\begin{array}{l}\text { 3-Methylglutaconic aciduria } \\
\text { type } 1\end{array}$ & 250950 & $A \cup H(A R)$ & $\begin{array}{l}\text { Carnitine supplements, avoid } \\
\text { fasting, emergency regimen }\end{array}$ & $\begin{array}{l}\text { Stabilizing/preventative } \\
\text { treatment }\end{array}$ & 5 & $d x . C P$ \\
\hline & Ethylmalonic encephalopathy & 602473 & ETHE1 (AR) & $\begin{array}{l}\mathrm{N} \text {-acetylcysteine, oral } \\
\text { metronidazol }\end{array}$ & $\begin{array}{l}\text { Stabilizing/preventative } \\
\text { treatment }\end{array}$ & 4 & $\begin{array}{l}\text { CNS malformations, episodic } \\
\text { ataxia; pyramidal tract signs }\end{array}$ \\
\hline & $\begin{array}{l}\text { Glutaric aciduria type I (GA1) } \\
\text { aka glutaryl-CoA dehydrogenase } \\
\text { deficiency }\end{array}$ & 231670 & $G C D H(A R)$ & $\begin{array}{l}\text { Lysine restriction, carnitine } \\
\text { supplements }\end{array}$ & $\begin{array}{l}\text { Stabilizing/preventative } \\
\text { treatment }\end{array}$ & $2 a$ & $\begin{array}{l}\text { Generalized spasticity, } \\
\text { dystonia with athethosis; } \\
\text { dx. CP; dyskinesia, dystonic } \\
\text { tetraparesis }\end{array}$ \\
\hline & Isovaleric acidemia & 243500 & IVD (AR) & $\begin{array}{l}\text { Dietary protein restriction, } \\
\text { carnitine supplements, avoid } \\
\text { fasting, emergency regimen }\end{array}$ & $\begin{array}{l}\text { Stabilizing/preventative } \\
\text { treatment }\end{array}$ & $2 c$ & Hypotonia, paresis \\
\hline & $\begin{array}{l}\text { Multiple acyl-CoA- } \\
\text { dehydrogenase deficiency } \\
\text { (MADD) (aka Glutaric aciduria } \\
\text { type II) }\end{array}$ & 231680 & $\begin{array}{l}\text { ETFA, ETFB, ETFDH } \\
(A R)\end{array}$ & $\begin{array}{l}\text { Carnitine, riboflavin, } \\
\beta \text {-hydroxybutyrate } \\
\text { supplements; emergency } \\
\text { regimen }\end{array}$ & $\begin{array}{l}\text { Primary/targeting } \\
\text { underlying } \\
\text { pathophysiology }\end{array}$ & 5 & Encephalopathy \\
\hline & Maple syrup urine disease & 248600 & $\begin{array}{l}D B T, B C K D H B, \\
B C K D H A(A R)\end{array}$ & $\begin{array}{l}\text { Dietary restriction, branched } \\
\text { amino-acids, avoid fasting, } \\
\text { (liver transplantation) }\end{array}$ & $\begin{array}{l}\text { Stabilizing/preventative } \\
\text { treatment (liver } t x= \\
\text { primary treatment) }\end{array}$ & $4(4)$ & $\begin{array}{l}\text { Spastic diplegic CP; } \\
\text { paroxysmal dystonia; ataxia }\end{array}$ \\
\hline & $\begin{array}{l}\text { Methylmalonic acidemia } \\
\text { (mutase deficiency) }\end{array}$ & 251000 & MUT (AR) & $\begin{array}{l}\text { Dietary protein restriction, } \\
\text { carnitine supplements, avoid } \\
\text { fasting, emergency regimen }\end{array}$ & $\begin{array}{l}\text { Stabilizing/preventative } \\
\text { treatment }\end{array}$ & $2 c$ & Total body dystonia \\
\hline & Lesch-Nyhan syndrome & 300322 & HPRT1 (X-linked) & $\begin{array}{l}\text { Haematopoietic stem cell } \\
\text { transplant }\end{array}$ & $\begin{array}{l}\text { Primary/targeting } \\
\text { underlying } \\
\text { pathophysiology }\end{array}$ & $4-5$ & $\begin{array}{l}\text { dx. Athetotic/dyskinetic CP; } \\
\text { dystonia }\end{array}$ \\
\hline
\end{tabular}


Table 2 Overview of all 54 treatable IEMs presenting as CP mimics identified through systematic literature review (Continued)

\begin{tabular}{|c|c|c|c|c|c|c|c|}
\hline & Propionic acidemia & 606054 & $P C C A, P C C B(A R)$ & $\begin{array}{l}\text { Dietary protein restriction, } \\
\text { carnitine supplements, } \\
\text { avoid fasting, emergency } \\
\text { regimen }\end{array}$ & $\begin{array}{l}\text { Stabilizing/preventative } \\
\text { treatment }\end{array}$ & $2 c$ & Dystonia, hypotonia \\
\hline \multirow[t]{4}{*}{ Urea cycle } & Argininemia & 207800 & $A R G 1(A R)$ & $\begin{array}{l}\text { Dietary protein restriction, } \\
\text { arginine supplement, sodium } \\
\text { benzoate, phenylbutyrate } \\
\text { (Liver transplantation) }\end{array}$ & $\begin{array}{l}\text { Stabilizing/preventative } \\
\text { treatment (liver } \mathrm{tx}= \\
\text { primary treatment) }\end{array}$ & $2 \mathrm{~b}(4)$ & $\begin{array}{l}\text { Spastic diplegia, ataxia, } \\
\text { dx. CP }\end{array}$ \\
\hline & Argininosuccinic aciduria & 207900 & $A S L(A R)$ & $\begin{array}{l}\text { Low protein diet, arginine- } \\
\text { supplements, sodium } \\
\text { benzoate, phenylbutyrate } \\
\text { (liver transplantation) }\end{array}$ & $\begin{array}{l}\text { Stabilizing/preventative } \\
\text { treatment (liver } \mathrm{tx}= \\
\text { primary treatment) }\end{array}$ & $2 b(4)$ & Cerebellar ataxia \\
\hline & Citrullinemia, type II & 605814 & SLC25A13 (AR) & $\begin{array}{l}\text { Dietary protein restriction, } \\
\text { arginine supplement, sodium } \\
\text { benzoate, phenylbutyrate } \\
\text { (Liver transplantation) }\end{array}$ & $\begin{array}{l}\text { Stabilizing/preventative } \\
\text { treatment (liver } t x= \\
\text { primary treatment) }\end{array}$ & $2 b(4)$ & dx. CP; spastic quadriplegia \\
\hline & $\begin{array}{l}\text { Ornithine transcarbamylase } \\
\text { deficiency }\end{array}$ & 311250 & OTC (X-linked) & $\begin{array}{l}\text { Dietary protein restriction, } \\
\text { citrulline supplements, sodium } \\
\text { benzoate/phenylbutyrate } \\
\text { (Liver transplantation) }\end{array}$ & $\begin{array}{l}\text { Stabilizing/preventative } \\
\text { treatment (liver } \mathrm{tx}= \\
\text { primary treatment) }\end{array}$ & $2 b(4)$ & $\begin{array}{l}\text { Hemiplegia; ataxia; gait } \\
\text { disturbance }\end{array}$ \\
\hline \multirow[t]{7}{*}{$\begin{array}{l}\text { Vitamins/ } \\
\text { Co-factors }\end{array}$} & Biotinidase deficiency & 2532760 & $B T D(A R)$ & Biotin supplement & $\begin{array}{l}\text { Primary/targeting } \\
\text { underlying } \\
\text { pathophysiology }\end{array}$ & $2 c$ & Spastic tetraparesis \\
\hline & $\begin{array}{l}\text { Biotin-thiamine-responsive } \\
\text { basal ganglia disease }\end{array}$ & 607483 & SLC19A3 (AR) & Biotin supplement & $\begin{array}{l}\text { Primary/targeting } \\
\text { underlying } \\
\text { pathophysiology }\end{array}$ & 4 & Ataxia, dystonia \\
\hline & $\begin{array}{l}\text { Cerebral folate deficiency } \\
\text { syndrome }\end{array}$ & 613068 & FOLR1 (AR) & Folinic acid & $\begin{array}{l}\text { Primary/targeting } \\
\text { underlying } \\
\text { pathophysiology }\end{array}$ & 4 & $\begin{array}{l}\text { spastic paraplegia; perinata } \\
\text { asphyxia }\end{array}$ \\
\hline & $\begin{array}{l}\text { Holocarboxylase synthetase } \\
\text { deficiency }\end{array}$ & 253270 & HLCS (AR) & Biotin supplement & $\begin{array}{l}\text { Primary/targeting } \\
\text { underlying } \\
\text { pathophysiology }\end{array}$ & 4 & dx. CP \\
\hline & $\begin{array}{l}\text { Hypermanganesemia with } \\
\text { dystonia, polycythemia, } \\
\text { and cirrhosis (HMDPC) }\end{array}$ & 613280 & SLC30A10 (AR) & Chelation therapy & $\begin{array}{l}\text { Primary/targeting } \\
\text { underlying } \\
\text { pathophysiology }\end{array}$ & 4 & Dystonia \\
\hline & $\begin{array}{l}\text { Molybdenum cofactor } \\
\text { deficiency }\end{array}$ & 252150 & $\begin{array}{l}\text { MOCS1, MOCS2, } \\
\text { (AR) }\end{array}$ & Precursor Z/cPMP & $\begin{array}{l}\text { Primary/targeting } \\
\text { underlying } \\
\text { pathophysiology }\end{array}$ & 4 & Spastic quadriplegia dx. CP \\
\hline & $\begin{array}{l}\text { Pyridoxamine } 5 \text { '-phosphate } \\
\text { oxidase deficiency }\end{array}$ & 610090 & PNPO (AR) & Pyridoxal 5'-phosphate & $\begin{array}{l}\text { Stabilizing/preventative } \\
\text { treatment }\end{array}$ & 4 & Spastic quadriplegia \\
\hline
\end{tabular}

Emergency regimen is defined as: Adjustment in management of a particular IEM to prevent or minimize metabolic decompensations (and related complications) during illness, periods of decreased intake or increased energy demand. The mainstay includes high caloric intake, generous fluid management (oral, tube or intravenous), addition/increase of vitamins/co-factors or medications, along with avoidance of substances which cannot be metabolized in patients with this IEM [24].

The IEMs are grouped according to the biochemical phenotype as presented in standard textbooks, and alphabetically. 


\begin{tabular}{|c|c|c|c|c|c|c|}
\hline Biochemical category & Disease name & OMIM\# & Gene(s) & Treatment & Effect & Level of evidence \\
\hline \multirow[t]{2}{*}{ Amino acids } & PSAT deficiency & 610992 & PSAT1 (AR) & $\begin{array}{l}\text { L-serine } \&+/ \text {-glycine } \\
\text { supplements }\end{array}$ & $\begin{array}{l}\text { Primary/targeting underlying } \\
\text { pathophysiology }\end{array}$ & 4 \\
\hline & $\begin{array}{l}\text { PSPH deficiency } \\
\text { (Serine deficiency) }\end{array}$ & 614023 & PSPH (AR) & $\begin{array}{l}\text { L-serine \& }+/ \text {-glycine } \\
\text { supplements }\end{array}$ & $\begin{array}{l}\text { Primary/targeting underlying } \\
\text { pathophysiology }\end{array}$ & 4 \\
\hline \multirow[t]{2}{*}{ Creatine } & $\begin{array}{l}\text { Arginine:glycine amidinotransferase } \\
\text { (AGAT) deficiency }\end{array}$ & 612718 & GATM (AR) & Creatine supplements & $\begin{array}{l}\text { Primary/targeting underlying } \\
\text { pathophysiology }\end{array}$ & 4 \\
\hline & Creatine transporter deficiency & 300352 & SLC6A8 (X-linked) & $\begin{array}{l}\text { Creatine, glycine, arginine } \\
\text { supplements }\end{array}$ & $\begin{array}{l}\text { Primary/targeting underlying } \\
\text { pathophysiology }\end{array}$ & 4 \\
\hline Fatty acid oxidation & $\begin{array}{l}\text { Carnitine palmitoyltransferase I } \\
\text { deficiency }\end{array}$ & 255120 & CPT1A (AR) & $\begin{array}{l}\text { Low-fat, high carbohydrate diet, } \\
\text { avoid fasting, Medium Chain } \\
\text { Triglyceride oil }\end{array}$ & $\begin{array}{l}\text { Stablizilng/preventative } \\
\text { treatment }\end{array}$ & 4 \\
\hline Hyperhomocystinuria & $\begin{array}{l}\text { Cobalamin deficiencies } \\
\text { (e.g., C, D, E, F, G) }\end{array}$ & $\begin{array}{l}251110,277400 \\
277410,236270 \\
277380\end{array}$ & $\begin{array}{l}\text { MMACHC, MMADHC, } \\
\text { MTRR, LMBRD1, MTR } \\
\text { (AR) }\end{array}$ & $\begin{array}{l}\text { Hydroxy-/cyanocobalamin } \\
\text { (+/- diet restriction, betaine, B12) }\end{array}$ & $\begin{array}{l}\text { Stabilizing/preventative } \\
\text { treatment }\end{array}$ & 4 \\
\hline Lipid storage (Leukodystrophy) & $\begin{array}{l}\text { Cerebrotendinous xanthomatosis } \\
\text { (CTX) }\end{array}$ & 213700 & CYP27A1 (AR) & Chenodeoxycholic acid & $\begin{array}{l}\text { Stabilizing/preventative } \\
\text { treatment }\end{array}$ & 4 \\
\hline \multirow[t]{3}{*}{ Organic acids } & HMG-CoA lyase deficiency & 246450 & HMGCL (AR) & $\begin{array}{l}\text { Protein restriction, avoid fasting, } \\
\text { emergency regimen }\end{array}$ & $\begin{array}{l}\text { Stabilizing/preventative } \\
\text { treatment }\end{array}$ & $4-5$ \\
\hline & mHMG-CoA synthase deficiency & 605911 & HMGCS2 (AR) & $\begin{array}{l}\text { Avoid fasting,emergency regimen, } \\
+/ \text {-dietary precursor restriction }\end{array}$ & $\begin{array}{l}\text { Stabilizing/preventative } \\
\text { treatment }\end{array}$ & 5 \\
\hline & SCOT deficiency & 245050 & OXCT1 (AR) & $\begin{array}{l}\text { Avoid fasting, protein restriction, } \\
\text { emergency regimen }\end{array}$ & $\begin{array}{l}\text { Stabilizing/preventative } \\
\text { treatment }\end{array}$ & 5 \\
\hline \multirow[t]{3}{*}{ Urea cycle } & $\begin{array}{l}\text { Carbamoyl phosphate synthetase } \\
\text { (CPS) deficiency }\end{array}$ & 237300 & CPS1 (AR) & $\begin{array}{l}\text { Dietary protein restriction, arginine } \\
\text { supplement, sodium benzoate, } \\
\text { phenylbutyrate (Liver transplantation) }\end{array}$ & $\begin{array}{l}\text { Stabilizing/preventative } \\
\text { treatment (primary/targeting } \\
\text { underlying pathophysiology) }\end{array}$ & $2 b(4)$ \\
\hline & Citrullinemia type I (ASS deficiency) & 215700 & ASS1 (AR) & $\begin{array}{l}\text { Dietary protein restriction, arginine } \\
\text { supplement, sodium benzoate, } \\
\text { phenylbutyrate (Liver transplantation) }\end{array}$ & $\begin{array}{l}\text { Stabilizing/preventative treatment } \\
\text { (primary/targeting underlying } \\
\text { pathophysiology) }\end{array}$ & $2 b(4)$ \\
\hline & $\begin{array}{l}\mathrm{N} \text {-acetyl-glutamate synthetase } \\
\text { deficiency }\end{array}$ & 237310 & NAGS (AR) & $\begin{array}{l}\text { Dietary protein restriction, arginine } \\
\text { supplement, sodium benzoate, } \\
\text { phenylbutyrate (Liver transplantation) }\end{array}$ & $\begin{array}{l}\text { Stabilizing/preventative } \\
\text { treatment }\end{array}$ & 4 \\
\hline
\end{tabular}

The IEMs are grouped according to the biochemical phenotype as presented in standard textbooks, and alphabetically. 
Table 4 Summary of all treatable IEMs $(n=38,57 \%)$ that can be detected by ' $1{ }^{\text {st }}$-tier' metabolic screening tests, which are affordable and accessible, with the potential to identify multiple IEMs

\begin{tabular}{|c|c|c|c|}
\hline \multicolumn{4}{|l|}{ Blood tests } \\
\hline \multirow[t]{3}{*}{ Acylcarnitine profiles $(n=3)$} & - MCAD deficiency & & \\
\hline & - SCAD deficiency & & \\
\hline & - VLCAD deficiency & & \\
\hline Free-to-total serum/plasma carnitine $(n=1)$ & $\begin{array}{l}\text { - Carnitine palmitoyltransferase I } \\
\text { deficiency }\end{array}$ & & \\
\hline \multirow[t]{4}{*}{ Plasma Amino Acids $(n=10)$} & - Argininemia & - Hartnup disease & - MTHFR Deficiency (\&tHcy) \\
\hline & $\begin{array}{l}\text { - Argininosuccinate lyase } \\
\text { deficiency }\end{array}$ & - HHH syndrome & $\begin{array}{l}\text { - Ornithine transcarbamylase } \\
\text { deficiency }\end{array}$ \\
\hline & - Citrullinemia type I & - Maple syrup urine disease & - Phenylketonuria (PKU) \\
\hline & - Citrullinemia, type II & & \\
\hline Plasma cholesterol $(n=1)$ & $\begin{array}{l}\text { - Cerebrotendinous xanthomatosis } \\
\text { (CTX) }\end{array}$ & & \\
\hline \multirow[t]{2}{*}{ Serum copper \& ceruloplasmin $(n=2)$} & $\begin{array}{l}\text { - Menkes Disease (\& urine } \\
\text { deoxypyridinoline) }\end{array}$ & & \\
\hline & - Wilson Disease (\& urine copper) & & \\
\hline \multicolumn{4}{|l|}{ Urine tests } \\
\hline Urine creatine metabolites $(n=1)$ & - GAMT deficiency & & \\
\hline Urine oligosaccharides $(n=1)$ & - Fucosidosis & & \\
\hline \multirow[t]{6}{*}{ Urine organic acids $(n=17)$} & - 3-Methylglutaconic aciduria type 1 & $\begin{array}{l}\text { - Ethylmalonic encephalopathy } \\
\text { (\& ACP) }\end{array}$ & - MHBD deficiency \\
\hline & $\begin{array}{l}\text { - 3-Methylcrotonyl-CoA carboxylase } \\
\text { (MCC) deficiency (\& ACP) }\end{array}$ & - SSADH & - HMG-CoA lyase deficiency \\
\hline & - $\beta$-Ketothiolase deficiency & - Glutaric aciduria type I & - mHMG-CoA synthase deficiency \\
\hline & - Cobalamin deficiencies (\& PAA) & $\begin{array}{l}\text { - Holocarboxylase synthetase } \\
\text { deficiency }\end{array}$ & $\begin{array}{l}\text { - Multiple acyl-CoA-dehydrogenase } \\
\text { deficiency (MADD) }\end{array}$ \\
\hline & - Cystathionine $\beta$-synthase deficiency & - Isovaleric academia & - Propionic academia \\
\hline & & - Methylmalonic academia & - SCOT deficiency \\
\hline \multirow[t]{2}{*}{ Urine purines \& pyrimidines $(n=2)$} & - Lesch-Nyhan syndrome & & \\
\hline & - Molybdenum cofactor deficiency & & \\
\hline
\end{tabular}

Abbreviations include: ACP acylcarnitine profiles, $t$ Hcy total homocystine, PAA plasma amnio acids.

expert opinion' (e.g., urine creatine metabolites identify GAMT deficiency, but also Creatine transporter deficiency and AGAT deficiency).

\section{Non-treatable IEMs}

There are several IEMs that can present as CP mimics that are not (yet) treatable. These include:

Disorders of amino acids (e.g., Hyperprolinemia type I); cholesterol (e.g., Mevalonic aciduria); lipids (e.g., FAHN, Pelizaeus-Merzbacher disease); lysosomal disorders, such as sphingolipidoses (e.g., GM1/2 gangliosidoses); mitochondrial diseases (e.g., Leigh's disease, sulfite oxidase deficiency, respiratory chain deficiencies); metals (e.g., NBIA1, PLAN); organic acids (e.g., Fumarate Hydratase Deficiency); peroxisomes (e.g., NALD); and purine and pyrimidine disorders (e.g., Adenylosuccinase deficiency, Purine nucleoside phosphorylase deficiency). Some of these conditions have emerging treatments, but not an established standard of care treatment; for example, Canavan disease, [25] and Gaucher disease, type 3 [26].

\section{Discussion}

To our knowledge, this is the first comprehensive literature review to extensively review and compiled all the known cases of treatable IEMs with co-occurring CP-like symptoms (dystonia, movement disorder, basal ganglia lesions, etc. before age 5 years). A surprisingly high number of $\mathrm{CP}$ mimics were identified, totaling 67 treatable IEMs (54 evidence-based, 13 expert-identified) and 43 non-treatable IEMs.

Among the treatable IEMs, we made the distinction between treatments that address primary causes of $\mathrm{CP}$ symptoms versus more secondary causes. For conditions that are primarily treatable, treatment targets the 
Table 5 All IEMs $(n=29,43 \%)$ requiring a specific ' 2 't - tier' test for diagnosis

\begin{tabular}{|c|c|c|}
\hline Biochemical category & Disease & Diagnostic test \\
\hline \multirow[t]{4}{*}{ Amino acids } & PSAT deficiency & CSF amino acids ( $\&$ PAA) \\
\hline & PSPH deficiency (Serine deficiency) & CSF amino acids ( \& PAA) \\
\hline & Late onset non-ketotic hyperglycinemia & CSF AA (\& Plasma AA) \\
\hline & PHGDH deficiency (Serine deficiency) & CSF AA (\& Plasma AA) \\
\hline Cerebral glucose transport & Blood brain-barrier glucose-transporter (GLUT1) defect & CSF glucose:plasma glucose ratio \\
\hline \multirow[t]{2}{*}{ Creatine } & Arginine: glycine amidinotransferase (AGAT) deficiency & GATM gene sequencing \\
\hline & Creatine transporter deficiency & SLC6A8 gene sequencing \\
\hline Lipids & Abetalipoproteinemia & $\begin{array}{l}\text { CBC smear, stool samples, fasting lipid profile, MTTP } \\
\text { gene analysis }\end{array}$ \\
\hline \multirow[t]{3}{*}{ Lysosomal } & Krabbe disease & WBC enzyme testing \\
\hline & Metachromatic leucodystrophy (MLD) & Arylsulfatase-A enzyme activity \\
\hline & Niemann-Pick, type C & $\begin{array}{l}\text { Filipin staining test (fibroblasts) \& NPC1/NPC2 gene } \\
\text { analyses }\end{array}$ \\
\hline \multirow[t]{3}{*}{ Mitochondria } & Coenzyme Q10 deficiency & Coenzyme Q10 (fibroblasts) \& gene(s) analysis \\
\hline & MELAS & Mitochondrial DNA mutation testing \\
\hline & Pyruvate dehydrogenase deficiency & $\begin{array}{l}\text { Blood \& CSF lactate:pyruvate ratio (enzyme activity, } \\
\text { gene(s) analysis) }\end{array}$ \\
\hline \multirow[t]{8}{*}{ Neurotransmission } & Aromatic-L-amino-acid decarboxylase deficiency & CSF biogenic amines \\
\hline & DHPR deficiency (biopterin deficiency) & CSF neurotransmitters \& biopterin loading test \\
\hline & Dopamine transporter deficiency syndrome & CSF neurotransmitters \\
\hline & GTPCH1-deficient dopa-responsive dystonia & $\begin{array}{l}\text { CSF neurotransmitters \& biopterin/Phe loading test; clinical } \\
\text { trial of L-dopamine, GTCPH gene analysis }\end{array}$ \\
\hline & PTPS deficiency (biopterin deficiency) & CSF neurotransmitters \& biopterin loading test \\
\hline & Sepiapterin reductase deficiency & CSF neurotransmitters \& biopterin/Phe loading test \\
\hline & Tyrosine hydroxylase deficiency & CSF neurotransmitters \& TH gene analysis \\
\hline & Vesicular monoamine transporter 2 (VMAT2) & CSF monoamine metabolites \\
\hline \multirow[t]{2}{*}{ Urea cycle } & Carbamoyl phosphate synthetase (CPS) deficiency & CPS gene analysis \\
\hline & N-acetyl-glutamate synthetase deficiency & NAGS gene analysis \\
\hline \multirow[t]{5}{*}{ Vitamins/Co-factors } & Biotinidase deficiency & Biotinidase enzyme activity \\
\hline & Biotin-thiamine-responsive basal ganglia disease & SLC19A3 gene analysis \\
\hline & Cerebral folate deficiency syndrome & CSF tetrahydrofolate \\
\hline & $\begin{array}{l}\text { Hypermanganesemia with dystonia, polycythemia, } \\
\text { and cirrhosis }\end{array}$ & $\begin{array}{l}\text { Whole-blood manganese concentrations, SLC30A10 gene } \\
\text { analysis }\end{array}$ \\
\hline & Pyridoxamine 5'-phosphate oxidase deficiency & Plasma, CSF \\
\hline
\end{tabular}

The IEMs are listed per biochemical category, with the specific biochemical/genetic diagnostic test per disease. Abbreviations include: CSF cerebrospinal fluid, PAA plasma amnio acids, Phe phenylalanine.

underlying pathophysiology and is most effective. For example, the neurotransmitter defect Tyrosine hydroxylase (TH) deficiency is highly amenable to early intervention treatment with L-dopa shows dramatic improvement and reversal of symptoms $[27,28]$. In diseases with secondary causes of CP symptoms (e.g., MCADD, MSUD, organic academia, urea cycle deficiency), metabolic crises such as hypoglycemia or acidosis caused by the metabolic defect can lead to neurologic sequelae mimicking CP. For these disorders prevention or stabilization, via emergency regimen, medical diets, etc., is best possible outcome.
Several IEMs presenting as CP mimics can be identified with minimally invasive testing. For example in biotinidase deficiency, the lack of the biotinidase enzyme causes accumulation of organic acid metabolites leading to ketolactic acidosis and hyperammonemia which can develop CPlike neurological manifestation (e.g., seisures, hypotonia, ataxia, feeding problems, cognitive developmental delay, etc.) [29]. Diagnosis requires minimally invasive testing (blood sampling for serum enzyme activity) and many of these symptoms can be alleviated following biotin supplementation and permanent neurological deficits 
such as optic atrophy, hearing loss and/or IDD may be prevented if treated early [29].

Non-treatable IEMs were also reported with the hope that new treatments might become available in the future. For example, experimental treatments are currently being explored in trials for Pantothenate kinase 2-associated neurodegeneration (PKAN, also known as HallervordenSpatz disease), such as gene therapy, chelation with Deferiprone [30] to prevent neurodegeneration caused by brain iron accumulation.

Although $48 \%$ of the IEMs listed can be identified by newborn screening (NBS) in most Canadian provinces [31], NBS is not universally standardized; also some diseases or very mild cases are missed. Therefore, these treatable IEMs should not be excluded from a differential diagnosis and are important to look for as part of clinical investigations for $\mathrm{CP}$. There are also be other non-IEM disorders that can present with CP symptoms (e.g. endocrine disorders [32]), which may be useful for the clinician to be aware.

Whole exome and genome sequencing allows for detection of new $\mathrm{CP}$ mimics and, along with other metabolomics approaches and enhanced neuroimaging, will facilitate research into the phenotypic spectrum and underlying pathophysiology of these disorders. In the future, screening for such conditions might be done by whole exome sequencing, with targeted analysis of the atypical $\mathrm{CP}$ genes, followed by biochemical confirmation for the IEMs listed here. However, it must be emphasized that clinical history and exam remain key in the interpretation of genomic data [33]. Furthermore the lumbar puncture, although invasive, should not be avoided as it allows for CSF neurotransmitter analysis, which is highly sensitive and often guides the clinician in further diagnostic and therapeutic decisions.

Despite our attempts to be as thorough as possible in this systematic review, we acknowledge the limitations of our study. Many of the IEMs listed are very rare diseases, with incidence ranging 1:10,000 (PKU) to 1:250,000 or less (GAMT deficiency), and thus, the number of publications is relatively low and evidence for treatments is sometimes sparse. As well, it can be difficult to publish case reports, which could contribute to a lack of literature evidence and preclude inclusion from our study. We have attempted to account for this by including expert clinician experience to identify IEMs that are not yet described in the literature. Despite our efforts to be as inclusive as possible when compiling the 'expert' list based on a working knowledge of IEMs that mimic CP (Table 3), we acknowledge that some of the potential candidates may have been omitted. Finally, neurologic symptoms are often insufficiently described in metabolic case reports, with focus often on the biochemical features of an IEM; this combined with the broad usage of the term of CP (and its different forms), the classification of an IEM phenotype as 'CP mimic' was challenging and depended on the authors' expertise.

The extensive number of distinct IEMs that may mimic $\mathrm{CP}$, each requiring particular diagnostic tests, places a significant information burden on clinicians. Here we have gone to extensive lengths to compile all known IEMs mimicking $\mathrm{CP}$ with the hope to help raise awareness and facilitate diagnostic approach with an established algorithm [19]. This is by no means meant as directive but rather, as a supportive tool to the clinician managing children with CP-like symptomatology. Symptoms which should prompt the clinician to search for an underlying IEM or other neurogenetic defect include -but not limited to- the following 'red flags': normal MRI findings imaging; abnormalities isolated to the globus pallidus; severe symptoms in the absence of a history of perinatal injury; a pattern of disease inheritance, or consanguinity; neurodevelopmental regression, or progressively worsening symptomatology; isolated muscular hypotonia; rigidity (as opposed to spasticity) on physician examination; paraplegia [33].

Early detection of treatable IEMs and timely intervention is of the utmost importance in order to prevent future brain insult and manifestation of CP symptoms. Additionally, the determination of the underlying cause of $\mathrm{CP}$, whether treatable or not, has significance from the point of view of risk assessment, counselling for families, improved access to community services, better management of co-morbidities, and the development of prevention and intervention strategies [13]. This would not only spare suffering of individuals, but would have broader impact in terms of alleviating the economic and social burden of $\mathrm{CP}$ as well.

As with the TIDE approach of systematic screening [19], it is our hope that the use of this algorithm will provide more insight into frequency of IEMs amongst the $\mathrm{CP}$ population, and further increase our understanding of the etiology of CP. Most importantly, early diagnosis of IEMs will allow initiation of causal treatment to improve outcomes via the reduction of possibly prevention of the the physical burdens of $\mathrm{CP}$.

\section{Conclusions}

We provided the first systematic review of treatable IEMs that can present with symptoms of CP. There are many single such reports in the literature, however, the collective incidence of treatable IEM mimicking $\mathrm{CP}$ is unknown and can be determined only by systematic or large-scale screening studies. Increasing clinician awareness might be worthwhile, as with timely diagnosis and appropriate treatment, these conditions can show improvement in the primary features, or stabilization and prevention of 
further neurologic sequelae and decline. The usefulness of our diagnostic algorithm remains to be determined but represents a first step towards increased recognition of potentially treatable conditions in the child with $\mathrm{CP}$.

\section{Additional file}

Additional file 1: Table S1. Overview of all 50 treatable IEMs

presenting as CP mimics identified through systematic literature review. The IEMs are grouped according to the biochemical phenotype as presented in standard textbooks, and alphabetically.

\section{Competing interests}

The authors declare that they have no competing interests.

\section{Authors' contributions}

ELL participated in study design, performed literature review and data collection, drafted the manuscript and performed revisions. MS provided clinical expertise and reviewed the manuscript. KB participated in study design, manuscript drafting and revision. SS provided clinical expertise, participated in the review of the literature and reviewed the manuscript. CDMvK conceived of, designed and supervised the study, reviewed literature results, provided clinical expertise, and participated in manuscript drafting and revision. All authors have read and approved the final manuscript.

\section{Authors' information}

ELL is a Genetic counselling student at the University of British Columbia (UBC). MS is pediatric neurologist in Montreal Children's Hospital, with special expertise in CP and IDD who has authored several guidelines and practice parameter; he is currently the Head of Pediatrics at McGill University. KB is a Post-doctoral fellow at the University of British Columbia. SS is a pediatric neurologist with expertise in neurometabolic diseases. She is head of the Biochemical Diseases division at BC Children's Hospital, Department of Pediatrics, UBC. She is the PI of the TIDE-BC study (www.tidebc.org). CDMvK is a Pediatrician-Biochemical Geneticist and Assistant Professor at the Department of Pediatrics, UBC. She has expertise in the diagnosis and discovery of treatable IEMs in intellectual disability and related disabilities; she is PI of the TIDEX gene discovery study at the Centre for Molecular Medicine and Therapeutics. The authors declare that they have no competing interests.

Sylvia Stockler and Clara D.M. van Karnebeek-www.tidebc.org and www.treatable-id.org.

\section{Acknowledgements}

This work was performed as part of the Treatable Intellectual Disability Endeavour in British Columbia (TIDE-BC, www.tidebc.org) funded by BC Children's Hospital as 1st Collaborative Area of Innovation. CVK is funded as a Michael Smith Foundation for Health Research Scholar. We gratefully acknowledge NeuroDevNet for input on the design of this study and their valued feedback.

\footnotetext{
Author details

${ }^{1}$ Provincial Medical Genetics Program, BC Women's Hospital, Vancouver, Canada. ${ }^{2}$ Division of Biochemical Diseases, Department of Pediatrics, BC Children's Hospital, University of British Columbia, Vancouver, Canada. ${ }^{3}$ Department of Pediatrics, Montreal Children's Hospital, McGill University, Montreal, Canada. ${ }^{4}$ Department of Neurology/Neurosurgery, Montreal Children's Hospital, McGill University, Montreal, Canada. ${ }^{5}$ Division of Biochemical Diseases, Department of Pediatrics, BC Children's Hospital, Vancouver, Canada. ${ }^{6}$ Treatable Intellectual Disability Endeavour in British Columbia, Vancouver, Canada. ${ }^{7}$ Centre for Molecular Medicine and Therapeutics; Child and Family Research Institute, Vancouver, Canada. ${ }^{8}$ Division of Biochemical Diseases, Department of Pediatrics, University of British Columbia, Rm K3-201, 4480 Oak Street, Vancouver, BC V6H 3V4, Canada.
}

Received: 12 September 2014 Accepted: 17 November 2014

Published online: 30 November 2014

\section{References}

1. Rosenbaum P, Paneth N, Leviton A, Goldstein M, Bax M, Damiano D, Dan B, Jacobsson B: A report: the definition and classification of cerebral palsy April 2006. Dev Med Child Neurol Supp/ 2007, 109:8-14.

2. Oskoui M, Coutinho F, Dykeman J, Jetté N, Pringsheim T: An update on the prevalence of cerebral palsy: a systematic review and meta-analysis. Dev Med Child Neurol 2013, 55(6):509-519.

3. Kirby RS, Wingate MS, Van Naarden BK, Doernberg NS, Arneson CL, Benedict RE, Mulvihill B, Durkin MS, Fitzgerald RT, Maenner MJ, Patz JA, Yeargin-Allsopp $M$ : Prevalence and functioning of children with cerebral palsy in four areas of the United States in 2006: a report from the Autism and Developmental Disabilities Monitoring Network. Res Dev Disabil 2011, 32(2):462-469.

4. Nelson KB: The epidemiology of cerebral palsy in term infants. Ment Retard Dev Disabil Res Rev 2002, 8(3):146-150.

5. Stanley FJ, Blair E: Why have we failed to reduce the frequency of cerebral palsy? Med J Aust 1991, 154(9):623-626.

6. Minear WL: A classification of cerebral palsy. Pediatrics 1956, 18(5):841-852.

7. Shevell MI, Dagenais L, Hall N, REPACQ Consortium: Comorbidities in cerebral palsy and their relationship to neurologic subtype and GMFCS level. Neurology 2009, 72(24):2090-2096.

8. Pakula AT, Van Naarden BK, Yeargin-Allsopp M: Cerebral palsy: classification and epidemiology. Phys Med Rehabil Clin N Am 2009, 20(3):425-452.

9. Parkes J, White-Koning M, Dickinson HO, Thyen U, Arnaud C, Beckung E, Fauconnier J, Marcelli M, McManus V, Michelsen SI, Parkinson K, Colver A: Psychological problems in children with cerebral palsy: a cross-sectional European study. J Child Psychol Psychiatry 2008, 49(4):405-413.

10. Dosa NP, Boeing NM, Ms N, Kanter RK: Excess risk of severe acute illness in children with chronic health conditions. Pediatrics 2001, 107(3):499-504.

11. Liptak GS, Shone LP, Auinger P, Dick AW, Ryan SA, Szilagyi PG: Short-term persistence of high health care costs in a nationally representative sample of children. Pediatrics 2006, 118(4):e1001-e1009.

12. Kancherla V, Amendah DD, Grosse SD, Yeargin-Allsopp M, Van Naarden BK Medical expenditures attributable to cerebral palsy and intellectual disability among Medicaid-enrolled children. Res Dev Disabil 2012, 33(3):832-840.

13. Ashwal S, Russman BS, Blasco PA, Miller G, Sandler A, Shevell M, Stevenson R, Quality Standards Subcommittee of the American Academy of Neurology: Practice Committee of the Child Neurology Society: Practice parameter: diagnostic assessment of the child with cerebral palsy: report of the Quality Standards Subcommittee of the American Academy of Neurology and the Practice Committee of the Child Neurology Society. Neurology 2004, 62(6):851-863.

14. Shevell M, Dagenais L, Oskoui M: The epidemiology of cerebral palsy: new perspectives from a Canadian registry. Semin Pediatr Neurol 2013, 20(2):60-64.

15. Saudubray JM, Desguerre I, Sedel F, Charpentier C: Acute symptoms in the neonatal period and early infancy. In A clinical approach to inherited metabolic diseases. Berlin Heidelberg: Springer; 2006:6-10.

16. Boyd K, Patterson V: Dopa responsive dystonia: a treatable condition misdiagnosed as cerebral palsy. Br Med J 1989, 298:1019-1020.

17. Nygaard TG, Waran SP, Levine RA, Naini AB, Chutorian AM: Dopa-responsive dystonia simulating cerebral palsy. Pediatr Neurol 1994, 11(3):236-240.

18. Longo N: Disorders of biopterin metabolism. J Inherit Metab Dis 2009, 32(3):333-342

19. van Karnebeek CD, Stockler S: Treatable inborn errors of metabolism causing intellectual disability: a systematic literature review. Mol Genet Metab 2012, 105(3):368-381.

20. van Karnebeek CDM, Shevell M, Zschocke J, Moeschler JB, Stockler S: The metabolic evaluation of the child with an intellectual developmental disorder: Diagnostic algorithm for identification of treatable causes and new digital resource. Mol Genet Metab 2014, 111(4):428-438.

21. Liberati A, Altman DG, Tetzlaff J, Mulrow C, Gøtzsche PC, loannidis JP, Clarke M, Devereaux PJ, Kleijnen J, Moher D: The PRISMA statement for reporting systematic reviews and meta-analyses of studies that evaluate healthcare interventions: explanation and elaboration. BMJ 2009, 339:b2700.

22. Gibson KM, Christensen E, Jakobs C, Fowler B, Clarke MA, Hammersen G, Raab K, Kobori J, Moosa A, Vollmer B, Rossier E, lafolla AK, Matern D, Brouwer OF, Finkelstein J, Aksu F, Weber HP, Bakkeren JA, Gabreels FJ, Bluestone D, Barron TF, Beauvais P, Rabier D, Santos C, Umansky R, Lehnert W: The clinical phenotype of succinic semialdehyde dehydrogenase deficiency (4-hydroxybutyric aciduria): case reports of 23 new patients. Pediatrics 1997, 99(4):567-574. 
23. Leuzzi V, Di Sabato ML, Deodato F, Rizzo C, Boenzi S, Carducci C, Malaspina P, Liberanome C, Dionisi-Vici C: Vigabatrin improves paroxysmal dystonia in succinic semialdehyde dehydrogenase deficiency. Neurology 2007, 68(16):1320-1321.

24. Dixon MA, Leonard JV: Intercurrent illness in inborn errors of intermediary metabolism. Arch Dis Child 1992, 67(11):387-1391.

25. Zano S, Malik R, Szucs S, Matalon R, Viola RE: Modification of aspartoacylase for potential use in enzyme replacement therapy for the treatment of Canavan disease. Mol Genet Metab 2011, 102(2):176-180.

26. Rice EO, Mifflin TE, Sakallah S, Lee RE, Sansieri CA, Barranger JA: Gaucher disease: studies of phenotype, molecular diagnosis and treatment. Clin Genet 1996, 49:111-118.

27. Furukawa Y, Graf WD, Wong H, Shimadzu M, Kish SJ: Dopa-responsive dystonia simulating spastic paraplegia due to tyrosine hydroxylase (TH) gene mutations. Neurology 2001, 56(2):260-263.

28. Hoffmann GF, Assmann B, Bräutigam C, Dionisi-Vici C, Häussler M, de Klerk JB, Naumann M, Steenbergen-Spanjers GC, Strassburg HM, Wevers RA: Tyrosine hydroxylase deficiency causes progressive encephalopathy and dopa-nonresponsive dystonia. Ann Neurol 2003, 54(Suppl 6):S56-S65

29. Wolf B: The neurology of biotinidase deficiency. Mol Genet Metab 2011 104(1-2):27-34.

30. Cossu G, Abbruzzese G, Matta G, Murgia D, Melis M, Ricchi V, Galanello R, Barella S, Origa R, Balocco M, Pelosin E, Marchese R, Ruffinengo U, Forni GL: Efficacy and safety of deferiprone for the treatment of pantothenate kinase-associated neurodegeneration (PKAN) and neurodegeneration with brain iron accumulation (NBIA): results from a four years follow-up. Parkinsonism Relat Disord 2014, 20(6):651-654.

31. Adams J: Newborn Screening in Canada Status Report. 2012. http://www. cadth.ca/products/environmental-scanning/environmental-scans/newbornscreening.

32. Tonduti $D$, Vanderver A, Berardinelli A, Schmidt JL, Collins CD, Novara F, Genni AD, Mita A, Triulzi F, Brunstrom-Hernandez JE, Zuffardi O, Balottin U, Orcesi S: MCT8 deficiency: extrapyramidal symptoms and delayed myelination as prominent features. J Child Neurol 2013, 28(6):795-800.

33. Lee RW, Poretti A, Cohen JS, Levey E, Gwynn H, Johnston MV, Hoon AH, Fatemi A: A diagnostic approach for cerebral palsy in the genomic era. Neuromolecular Med. in press.

doi:10.1186/s13023-014-0197-2

Cite this article as: Leach et al:: Treatable inborn errors of metabolism presenting as cerebral palsy mimics: systematic literature review.

Orphanet Journal of Rare Diseases 2014 9:197.

\section{Submit your next manuscript to BioMed Central and take full advantage of:}

- Convenient online submission

- Thorough peer review

- No space constraints or color figure charges

- Immediate publication on acceptance

- Inclusion in PubMed, CAS, Scopus and Google Scholar

- Research which is freely available for redistribution 